\title{
Probing neutron star physics using accreting neutron stars
}

\author{
Alessandro Patruno ${ }^{\mathrm{a}}$ \\ Astronomical Institute "Anton Pannekoek", University of Amsterdam, 1098XH Amsterdam, The Netherlands
}

\begin{abstract}
We give an obervational overview of the accreting neutron stars systems as probes of neutron star physics. In particular we focus on the results obtained from the periodic timing of accreting millisecond X-ray pulsars in outburst and from the measurement of X-ray spectra of accreting neutron stars during quiescence. In the first part of this overview we show that the $\mathrm{X}$-ray pulses are contaminated by a large amount of noise of uncertain origin, and that all these neutron stars do not show evidence of spin variations during the outburst. We present also some recent developments on the presence of intermittency in three accreting millisecond X-ray pulsars and investigate the reason why only a small number of accreting neutron stars show X-ray pulsations and why none of these pulsars shows sub-millisecond spin periods. In the second part of the overview we introduce the observational technique that allows the study of neutron star cooling in accreting systems as probes of neutron star internal composition and equation of state. We explain the phenomenon of the deep crustal heating and present some recent developments on several quasi persistent X-ray sources where a cooling neutron star has been observed.
\end{abstract}

The participation at this summer school was supported by the HISS Dubna program of the Helmholtz association and by CompStar, a Research Networking Programme of the European Science Foundation.

\section{References}

Romanova et al.(2008). Romanova, M. M., Long, M., Kulkarni, A. K., Kurosawa, R., Ustyugova, G. V., Koldoba, A. K., \& Lovelace, R. V. E. 2008, arXiv:0803.2865

Long et al.(2008). Long, M., Romanova, M. M., \& Lovelace, R. V. E. 2008, MnRas, 386, 1274

Patruno et al.(2009). Patruno, A., Wijnands, R., \& van der Klis, M. 2009, ApJL, 698, L60

Watts et al.(2008). Watts, A. L., Patruno, A., \& van der Klis, M. 2008, ApJL, 688, L37

Hartman et al.(2008). Hartman, J. M., et al. 2008, ApJ , 675, 1468

Altamirano et al.(2008). Altamirano, D., Casella, P., Patruno, A., Wijnands, R., \& van der Klis, M. 2008, ApJL, 674, L45

Casella et al.(2008). Casella, P., Altamirano, D., Patruno, A., Wijnands, R., \& van der Klis, M. 2008, ApJL, 674, L41

Lamb et al.(2008). Lamb, F. K., Boutloukos, S., Van Wassenhove, S., Chamberlain, R. T., Lo, K. H., \& Miller, M. C. 2008, arXiv:0809.4016

Galloway et al.(2007). Galloway, D. K., Morgan, E. H., Krauss, M. I., Kaaret, P., \& Chakrabarty, D. 2007, ApJL , 654, L73

Wijnands \& van der Klis(1998). Wijnands, R., \& van der Klis, M. 1998, Nature , 394, 344

Hessels et al.(2006). Hessels, J. W. T., Ransom, S. M., Stairs, I. H., Freire, P. C. C., Kaspi, V. M., \& Camilo, F. 2006, Science, 311,1901

\footnotetext{
a e-mail: a.patruno@uva.nl
}

Cumming et al.(2001). Cumming, A., Zweibel, E., \& Bildsten, L. 2001, ApJ , 557, 958

Yakovlev \& Pethick(2004). Yakovlev, D. G., \& Pethick, C. J 2004, ARAA , 42, 169

Page et al.(2006). Page, D., Geppert, U., \& Weber, F. 2006, Nuclear Physics A, 777, 497

Cackett et al.(2008). Cackett, E. M., Wijnands, R., Miller, J. M., Brown, E. F., \& Degenaar, N. 2008, ApJL, 687, L87

Cackett et al.(2006). Cackett, E. M., Wijnands, R., Linares, M., Miller, J. M., Homan, J., \& Lewin, W. H. G. 2006, MnRas , 372,479

Wijnands(2006). Wijnands, R. 2006, Trends in Pulsar Research, 53

Fröhlich(2003). Fröhlich, H.-E. 2003, Sterne und Weltraum, 42, 100000

Rutledge et al.(2002). Rutledge, R. E., Bildsten, L., Brown, E. F., Pavlov, G. G., Zavlin, V. E., \& Ushomirsky, G. 2002, ApJ , 580, 413

Brown et al.(1998). Brown, E. F., Bildsten, L., \& Rutledge, R. E. 1998, ApJL, 504, L95

Shternin et al.(2007). Shternin, P. S., Yakovlev, D. G., Haensel, P., \& Potekhin, A. Y. 2007, MnRas, 382, L43

Heinke et al.(2007). Heinke, C. O., Jonker, P. G., Wijnands, R., \& Taam, R. E. 2007, ApJ , 660, 1424

This is an Open Access article distributed under the terms of the Creative Commons Attribution-Noncommercial License 3.0, which permits unrestricted use, distribution, and reproduction in any noncommercial medium, provided the original work is properly cited. 


\title{
Probing the neutron star physics with accreting neutron stars (part 1)
}

\author{
Alessandro Patruno \\ University of Amsterdam \\ The Netherlands
}

\section{Lecture 1: outline}

- Some refreshment on X-ray binaries

- Measure of the spin period (part 1)

- Measure of the spin torque of the NS

- Measure of the spin period (part 2)

- Why only 10 LMXB pulsate ?

- Do submillisecond pulsars exist?

- Measure of the mass 


\section{How to probe the NS physics with NS LMXBs?}

1. X-ray spectra (cooling, cyclotron resonance, etc...)

2. Coherent timing (pulse profile shape, torques, timing noise, mass, glitches)

3. Thermonuclear bursts

4. Aperiodic variability (oscillation modes, QPOs)

Use of three wonderful satellites: Chandra, XMM-Newton, RXTE, Suzaku, Swift
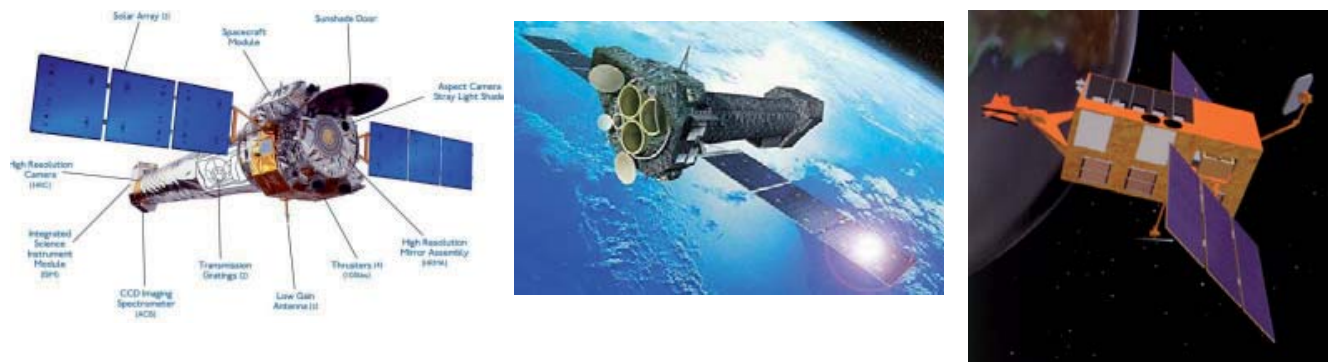

\section{X-ray binaries: the Roche potential

Any gas flow between two stars is governed by the Euler equation (conservation of momentum for each gas element):

$$
\rho \frac{\partial \vec{v}}{\partial t}+\rho \vec{v} \cdot \nabla \vec{v}=-\nabla P+\vec{f}
$$

In the co-rotating reference
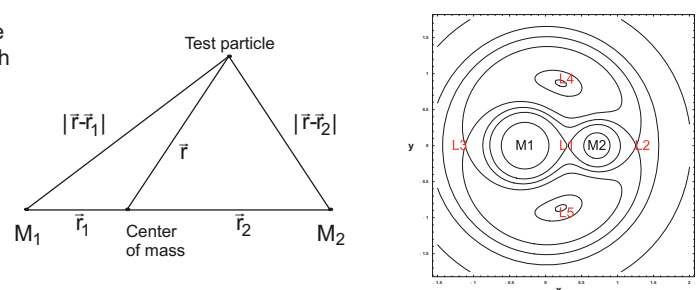

frame of a binary it becomes:
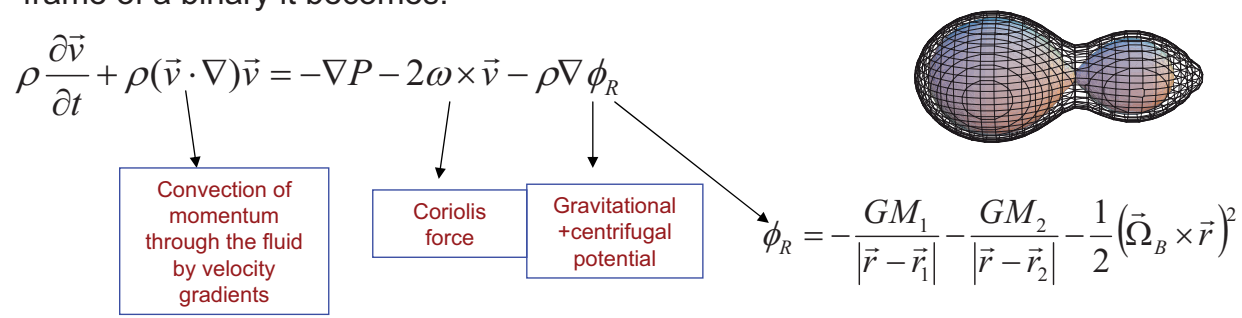


\section{The family of NS X-ray binaries}

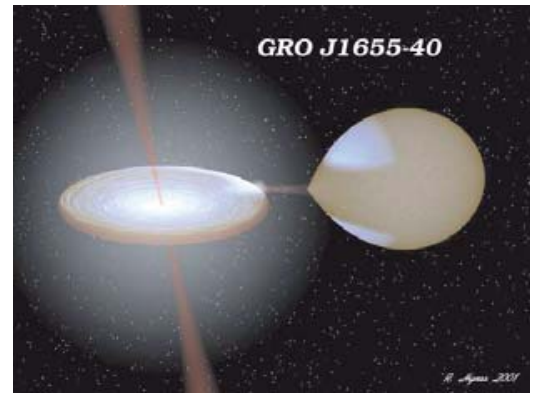

Low mass X-ray binaries

- Roche lobe overflow

- low mass companions

- old NSs

- accretion driven by an accretion disc

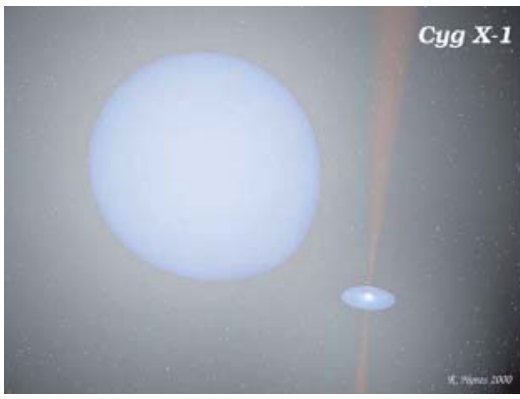

High mass X-ray binaries

- Wind fed accretion

- high mass companions

- young NSs

- a disc not always can form

\section{Transient LMXBs}

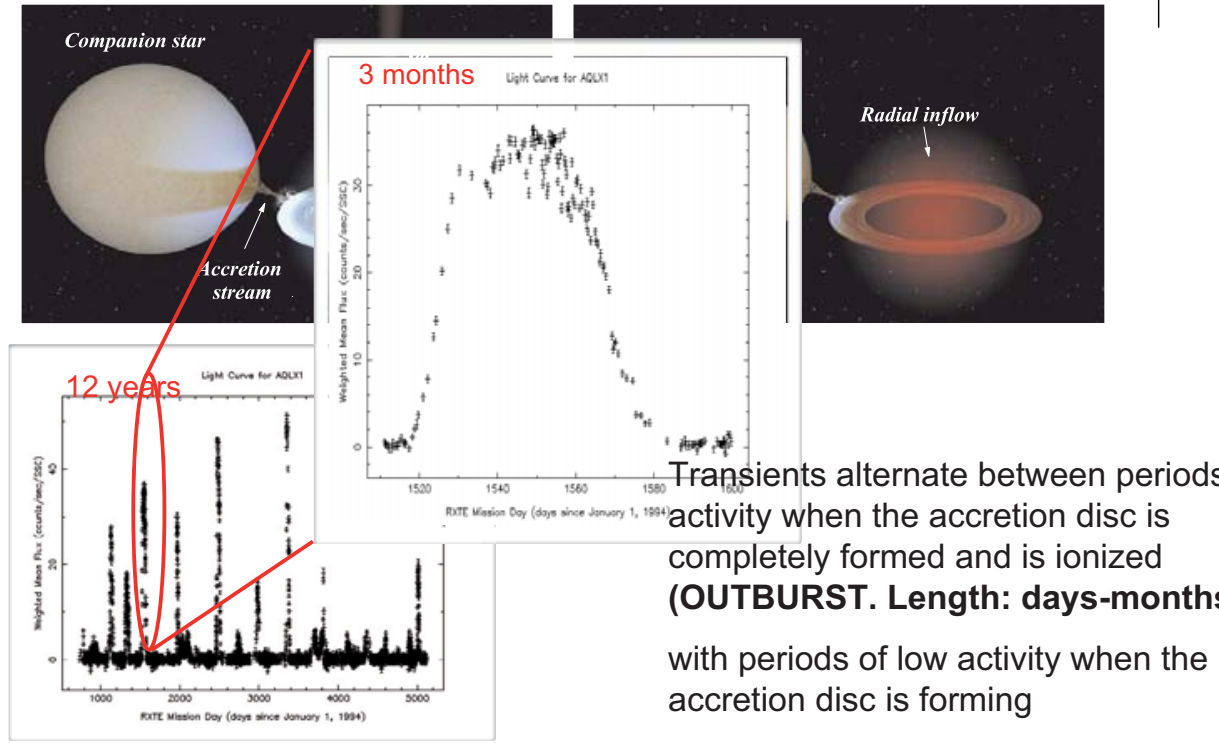

(QUIESCENCE. Length: months-years) 


\section{Low mass X-ray binaries}
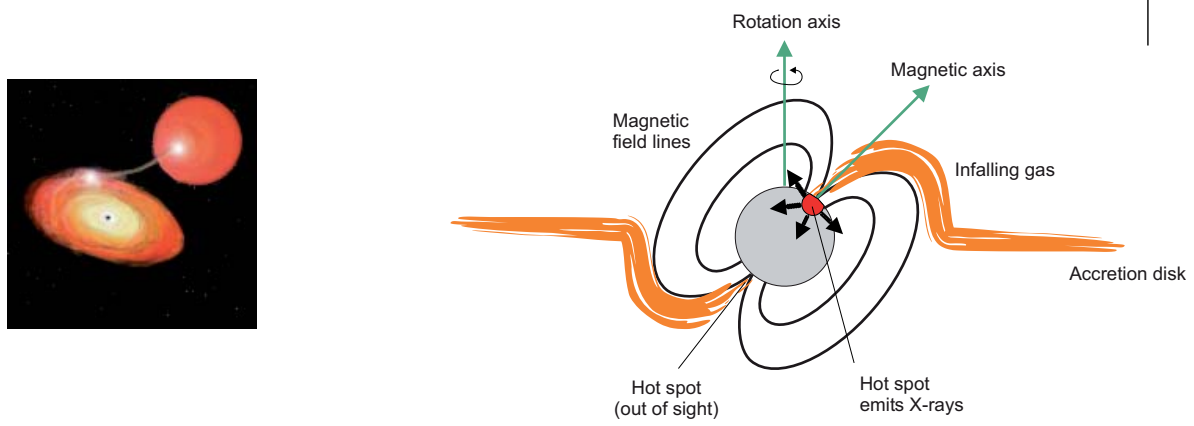

Conservation of angular momentum and viscosity leads to the formation of an accretion disc. The gas flows in the inner part of the primary Roche lobe till the following condition holds:

$$
P_{\text {mag }}=\frac{B^{2}}{8 \pi}>>\left(P_{\text {gas }}, P_{\text {ram }}\right)
$$

The gas then flows along the B filed lines and hits the NS surface

$$
L_{E d d} \approx 1.3 \times 10^{38}\left(\frac{M}{M_{\text {Sun }}}\right) \mathrm{erg} / \mathrm{s}
$$

\section{Accreting millisecond pulsars}

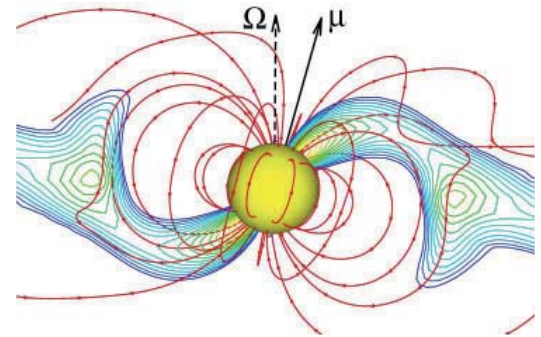

$$
\begin{aligned}
& R_{A}=\left(\frac{2 \mu^{2} G^{2} M_{N S}^{2}}{\dot{M}_{c}}\right) \propto M_{N S}^{1 / 7} R^{-2 / 7} L^{-2 / 7} \mu^{4 / 7} \\
& R_{c o}=\left(\frac{G M_{N S}}{\omega^{2}}\right)^{1 / 3} \approx 2.8 \times 10^{3} M_{N S}^{1 / 3} P_{s}^{1 / 2} K m
\end{aligned}
$$

$$
R_{A}<R_{c o} \longrightarrow \quad \begin{gathered}
\begin{array}{c}
\text { Accretion is possible. Plasma follows the field } \\
\text { line of the NS magnetic field }
\end{array} \\
\text {. }
\end{gathered}
$$

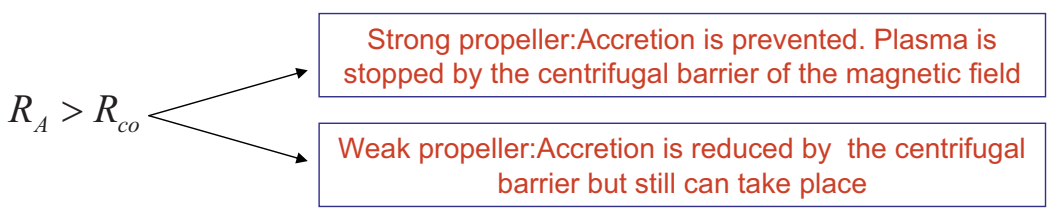




\section{The funnel stream}

The green surface is a constant density surface, and red lines are sample magnetic field lines. Funnel streams hit the surface of the star at approximately the same position at all times, creating quasi-stationary hot spots.

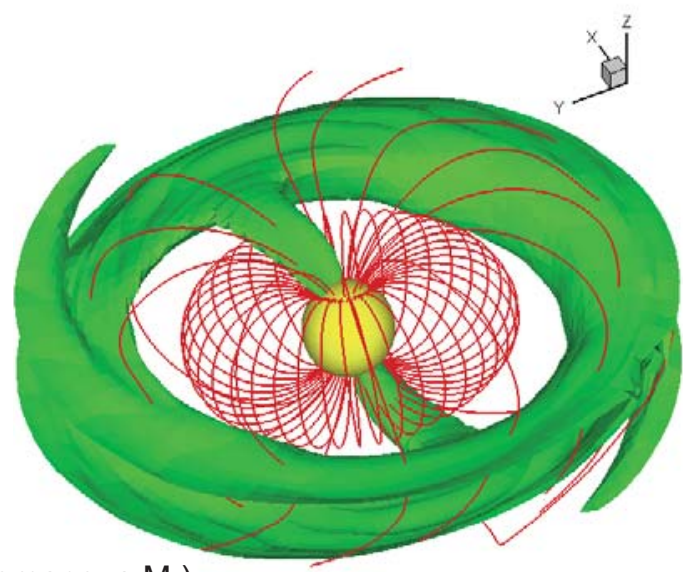

Animation from the Cornell group (Romanova M.)

http://www.astro.cornell.edu/us-russia/propeller.htm

\section{Accreting millisecond pulsars}

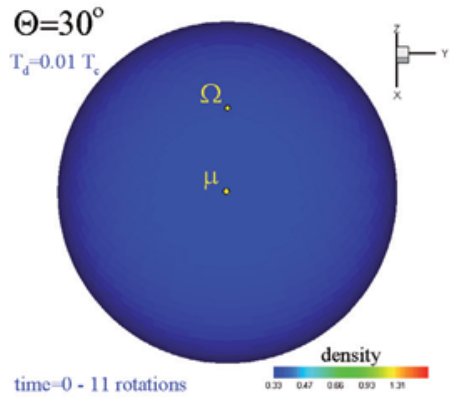

The "hot spot" created during accretion can move around the NS surface and is not completely locked to the poles.

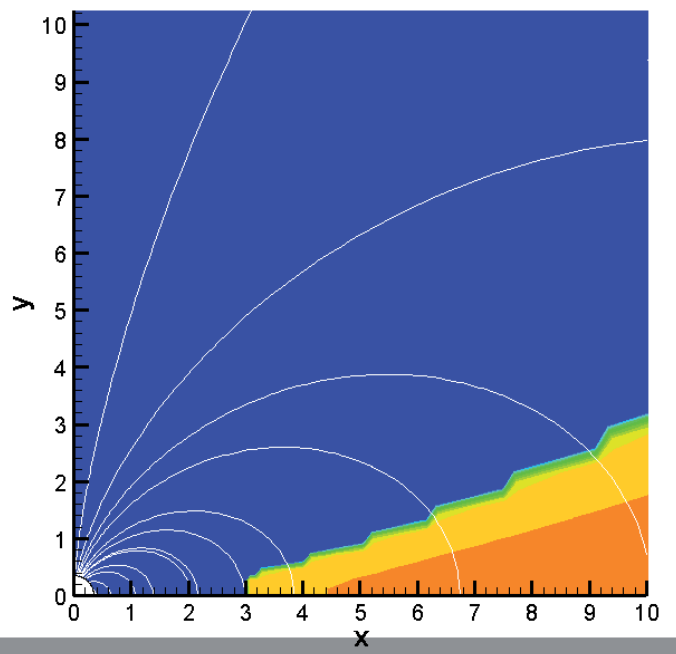

Animation from the Cornell group (Romanova M.)

http://www.astro.cornell.edu/us-russia/propeller.htm 


\section{How to create a sinusoidal profile}

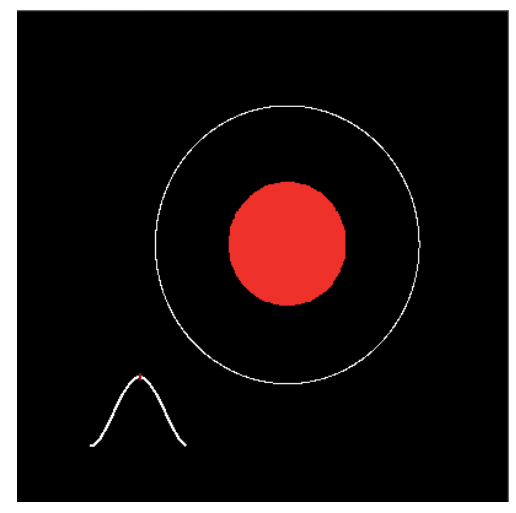

Animation from F. Ozel:

http://www.physics.arizona.edu/ fozel/

GR and SR effects are important here!

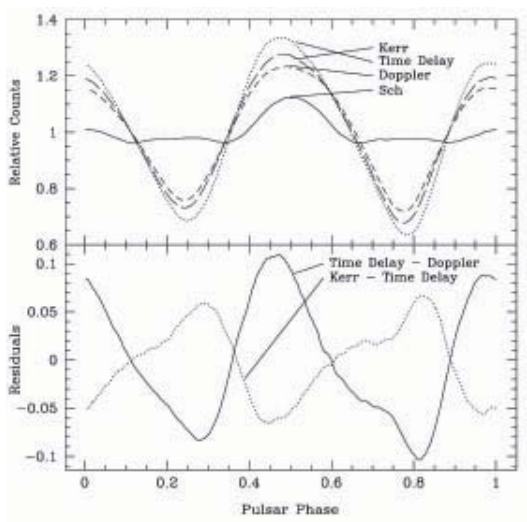

\section{The measure of the spin period (part 1)}




\section{Observations: the lightcurves}

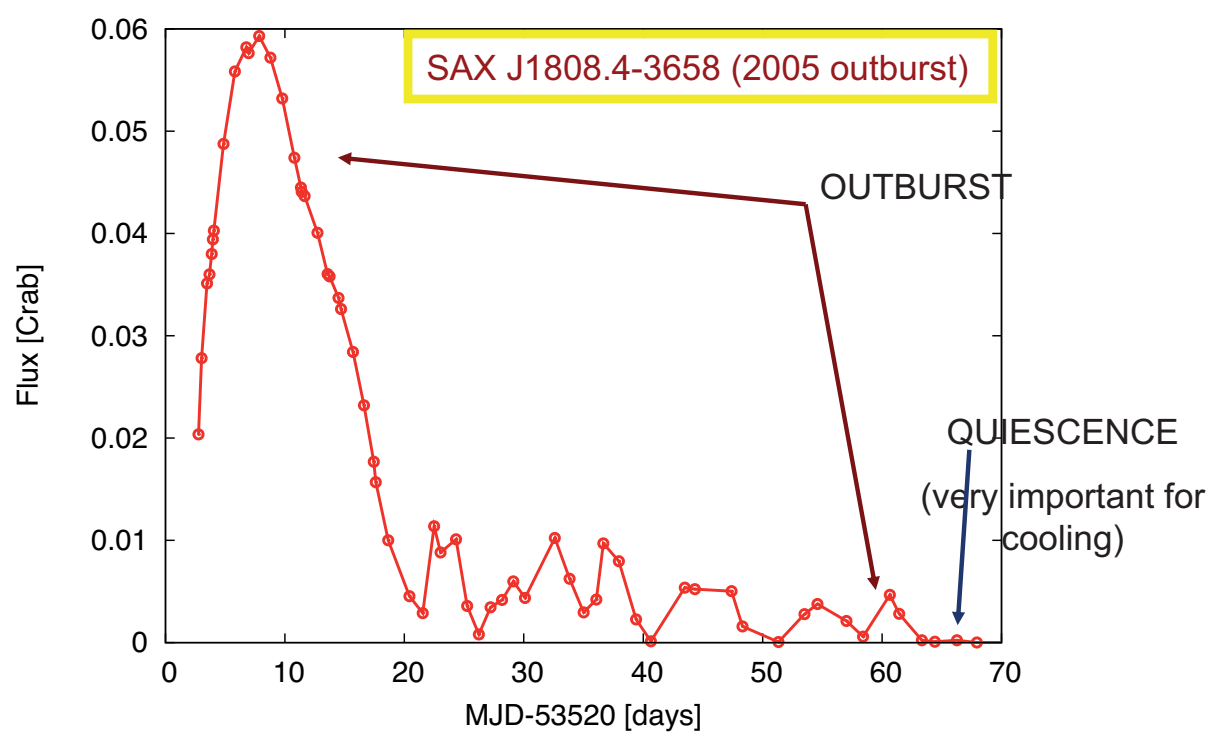

SAX J1808.4-3658

A clear spike emerges in the PDS of the lightcurve.

The spike is at the spin frequency of the neutron star.

Folding the data (to increase the $\mathrm{S} / \mathrm{N}$ ) at the spin frequency creates the average pulse profile 


\section{The AMXPs family}

\begin{tabular}{|c|c|c|c|}
\hline Name & Spin frequency [Hz] & Orbital Period [hr] & Reference \\
\hline SAX J1808.4-3658 & 401 & 2.1 & $\begin{array}{c}\text { Wijnands \& van der Klis } \\
\text { (1998) } \\
\text { Chakrabarty \& Morgan } \\
(1998)\end{array}$ \\
\hline XTE J1751-305 & 435 & 0.70 & Markwardt et al. 2002 \\
\hline XTE J0929-314 & 185 & 0.73 & Galloway et al. 2002 \\
\hline XTE J1807-294 & 190 & 0.67 & Markwardt et al. 2003 \\
\hline XTE J1814-334 & 314 & 4 & Markwardt et al. 2003 \\
\hline IGR J00291+5934 & 599 & 2.5 & Galloway et al. 2005 \\
\hline SWIFT J1756.9-2508 & 180 & 0.90 & Markwardt et al. 2007 \\
\hline
\end{tabular}

\section{Measured spin torques}

\begin{tabular}{|c|c|c|c|}
\hline Name & $\begin{array}{c}\text { Spin frequency } \\
{[\mathrm{Hz}]}\end{array}$ & $\begin{array}{c}\text { Spin torque } \\
{[1 \mathrm{E}-13 \mathrm{~Hz} \text { s] }}\end{array}$ & Reference \\
\hline SAX J1808.4-3658 & 401 & $\begin{array}{c}4.4(0.83) \\
-0.76(0.23) \\
<|0.25|\end{array}$ & $\begin{array}{c}\text { Burderi et al.(2006) } \\
\text { Hartman et al.(2008) }\end{array}$ \\
\hline XTE J1751-305 & 435 & $3.7(1.0)$ & Papitto et al. (2008) \\
\hline XTE J0929-314 & 185 & $-0.92(0.40)$ & Galloway et al. (2002) \\
\hline XTE J1807-294 & 190 & $0.25(0.10)$ & $\begin{array}{c}\text { Riggio et al. (2008) } \\
\text { Patruno et al. (2008) }\end{array}$ \\
\hline XTE J1814-334 & 314 & $-0.67(0.07)$ & $\begin{array}{c}\text { Papitto et al. (2007) } \\
\text { Watts, Patruno } \& \text { van der } \\
\text { Klis (2008) }\end{array}$ \\
\hline IGR J00291+5934 & 599 & $\begin{array}{c}8.4(0.6) \\
8.5(1.1)\end{array}$ & $\begin{array}{c}\text { Falanga et al. (2005) } \\
\text { Burderi et al. (2007) }\end{array}$ \\
\hline SWIFT J1756.9-2508 & 180 & $X X$ & \\
\hline
\end{tabular}




\section{Pulse profiles}
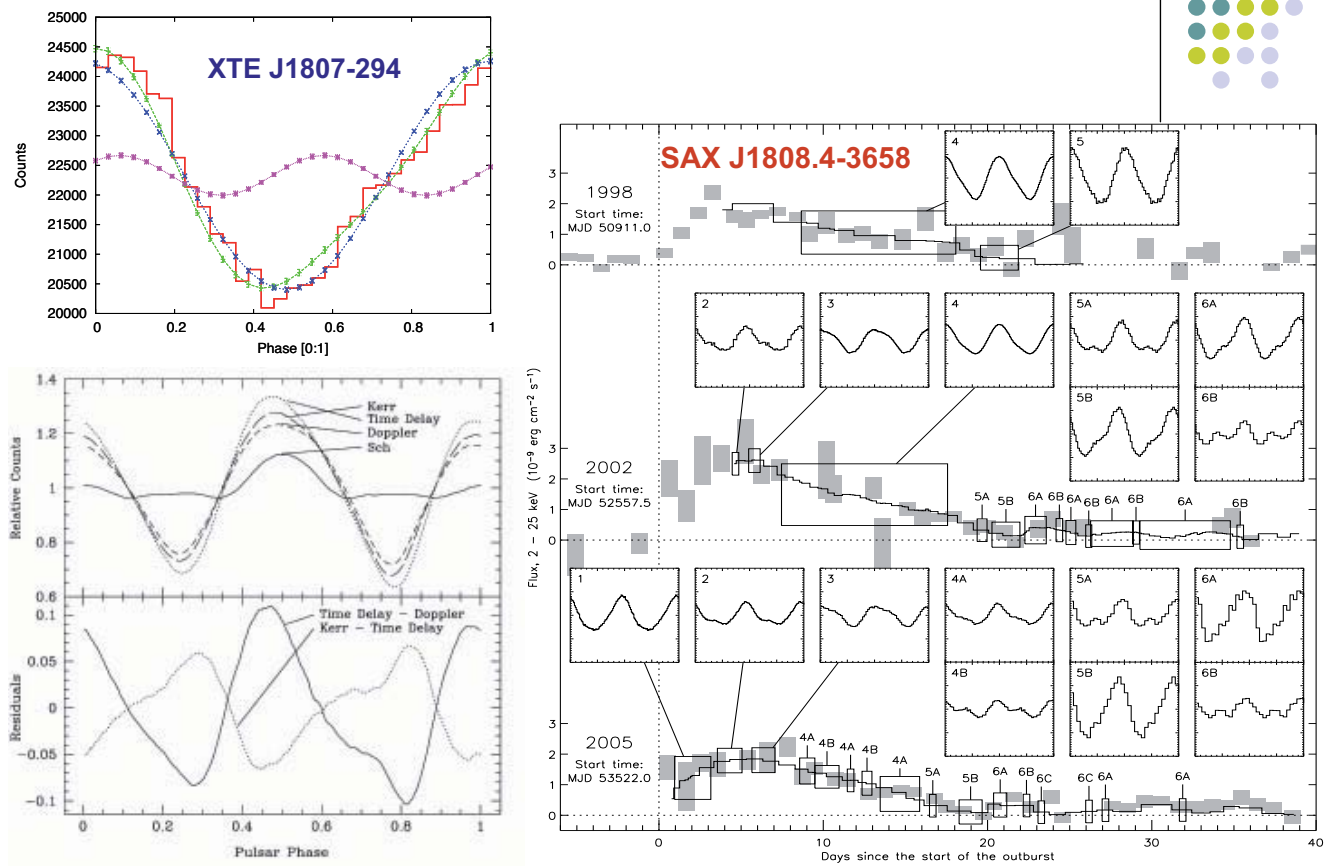

\section{The Harmonic decomposition}

- Assume uncorrelated noise in the pulse TOA uncertainities (least-squares algorithm)

- Decompose the pulse profiles in their sinusoidal components: $y=A \sin \left(\omega t+\phi_{1}\right)+B \sin \left(2 \omega t+\phi_{2}\right)+C$

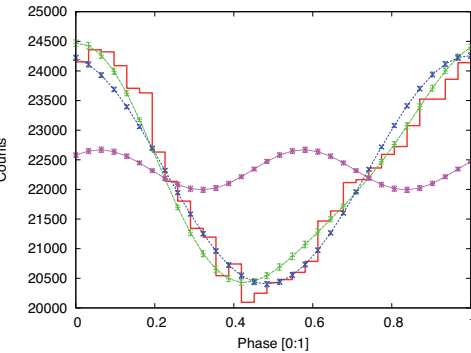

1st harmonic $\quad v=v_{\text {spin }}$

$2^{\text {nd }}$ harmonic $v=2 v_{\text {spin }}$

Fit the phases with a polynomial expansion

$$
\phi=\phi_{0}+v\left(t-t_{0}\right)+\frac{1}{2} \dot{v}\left(t-t_{0}\right)^{2}+\ldots
$$



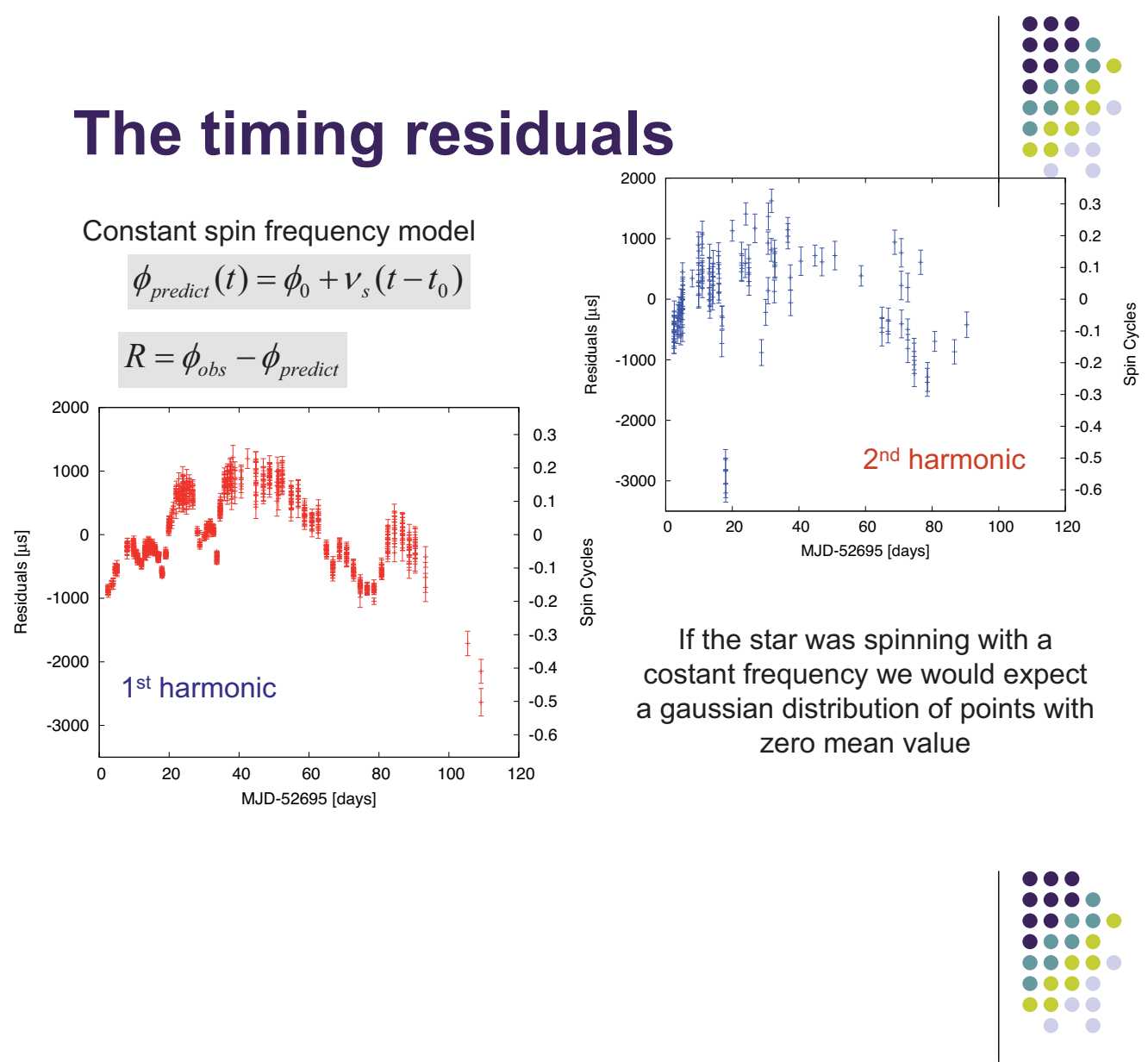

\section{The measure of the spin torque}


SAX J1808.4-3658: do we really observe a spin torque ?
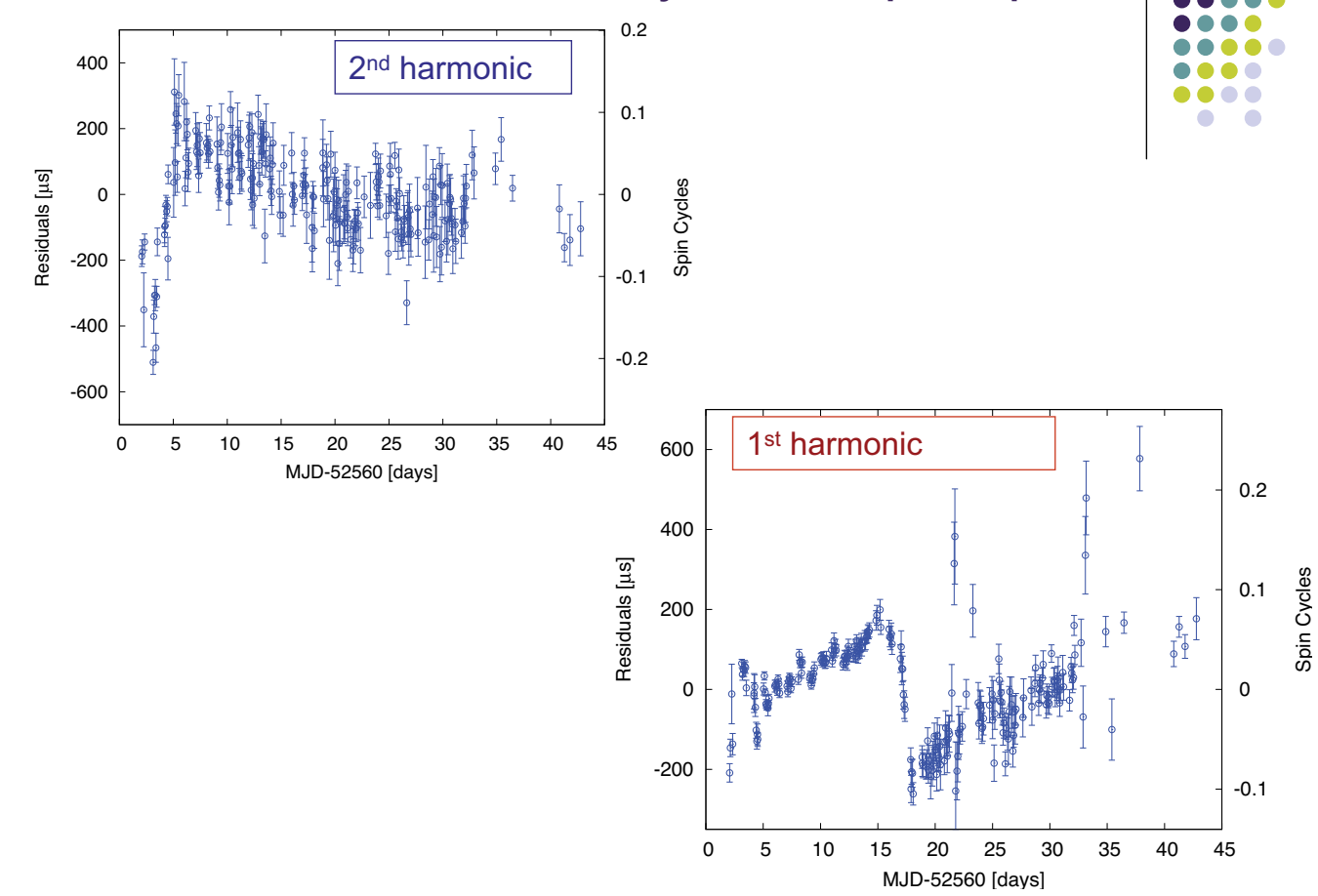

\section{To spin or not to spin?}

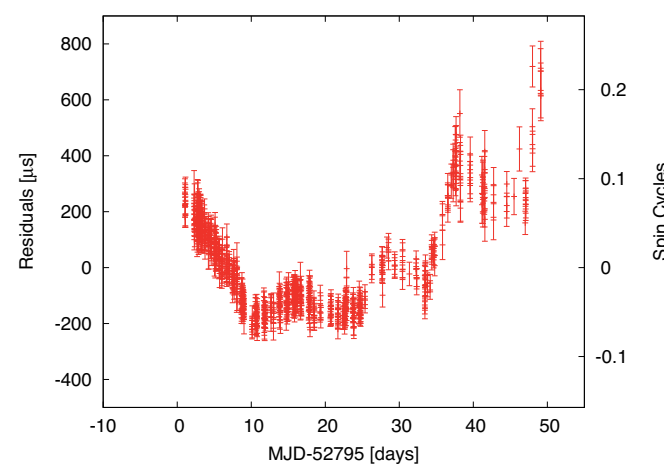

\begin{tabular}{|c|c|}
\hline AMXP & Noise level \\
\hline SAX J1808.4-3658 & High \\
\hline XTE J1751-305 & Low \\
\hline XTE J0929-314 & Very low \\
\hline XTE J1807-294 & Very high \\
\hline XTE J1814-334 & High \\
\hline IGR J00291+5934 & Low \\
\hline SWIFT J1756.9-2508 & $x x$ \\
\hline
\end{tabular}

Basically all the AMXPs show "timing noise" at some degree.

What is the origin of this 'noise'?

Noise is does not mean "measurement noise" (boring) but some unknown origin of the phenomenon. Can be hiding the best part of the physics there! 


\section{The origin of "timing noise"}

Timing noise might be the most important and interesting part of the NS physics. It's not just a 'measurement noise' !!!

1. Transfer of angular momentum

2. Superfluidity

3. Magnetic field

4. Accretion process and disc-magnetosphere interaction

It is observed in: radio pulsar (young), magnetars, HMXBs, LMXBs (both AMXPs and slowly rotating)

\section{Why the number of pulsating LMXBs is so small?}




\section{Why not all the NS-LMXBs pulsate?}

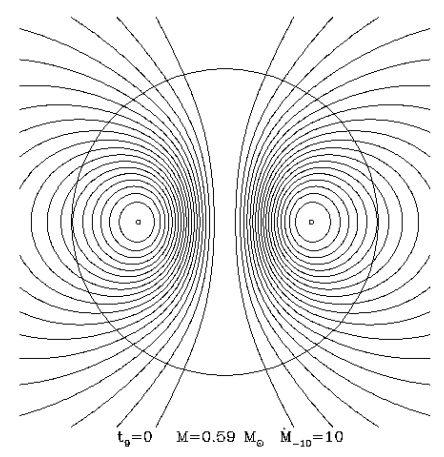

(Animation: Andrew Cumming)
The freshly accreted diamagnetic material destroys the external $B$ field.

The Ohmic diffusion on the contrary tries to magnetize the accreted material.

\section{Intermittent pulsar 1: HETE J1900+2455}

This source was behaving like a normal AMXPs, then the pulsations disappeared after $\sim 2$ months.

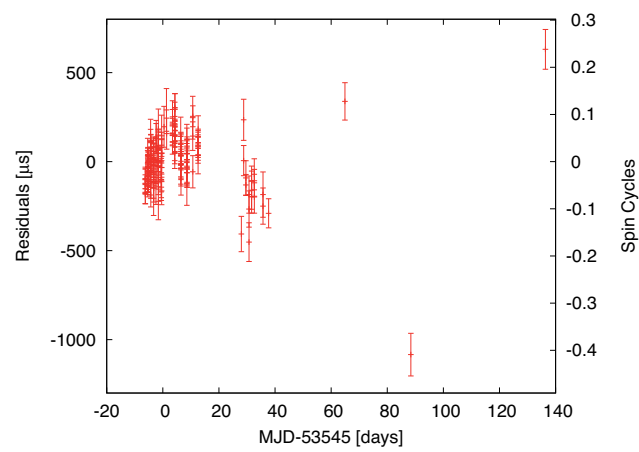

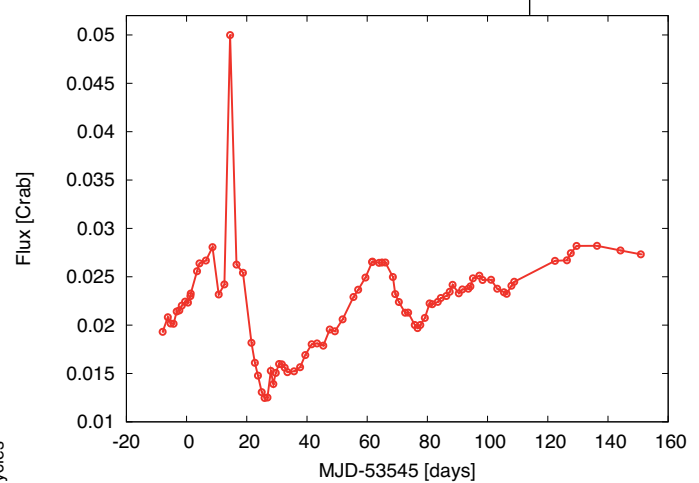

Pulsation at $\sim 377 \mathrm{~Hz}$

Pulsations in $\sim 10 \%$ of the exposure 


\section{Intermittent pulsar 2: SAX J1748.9-2021}
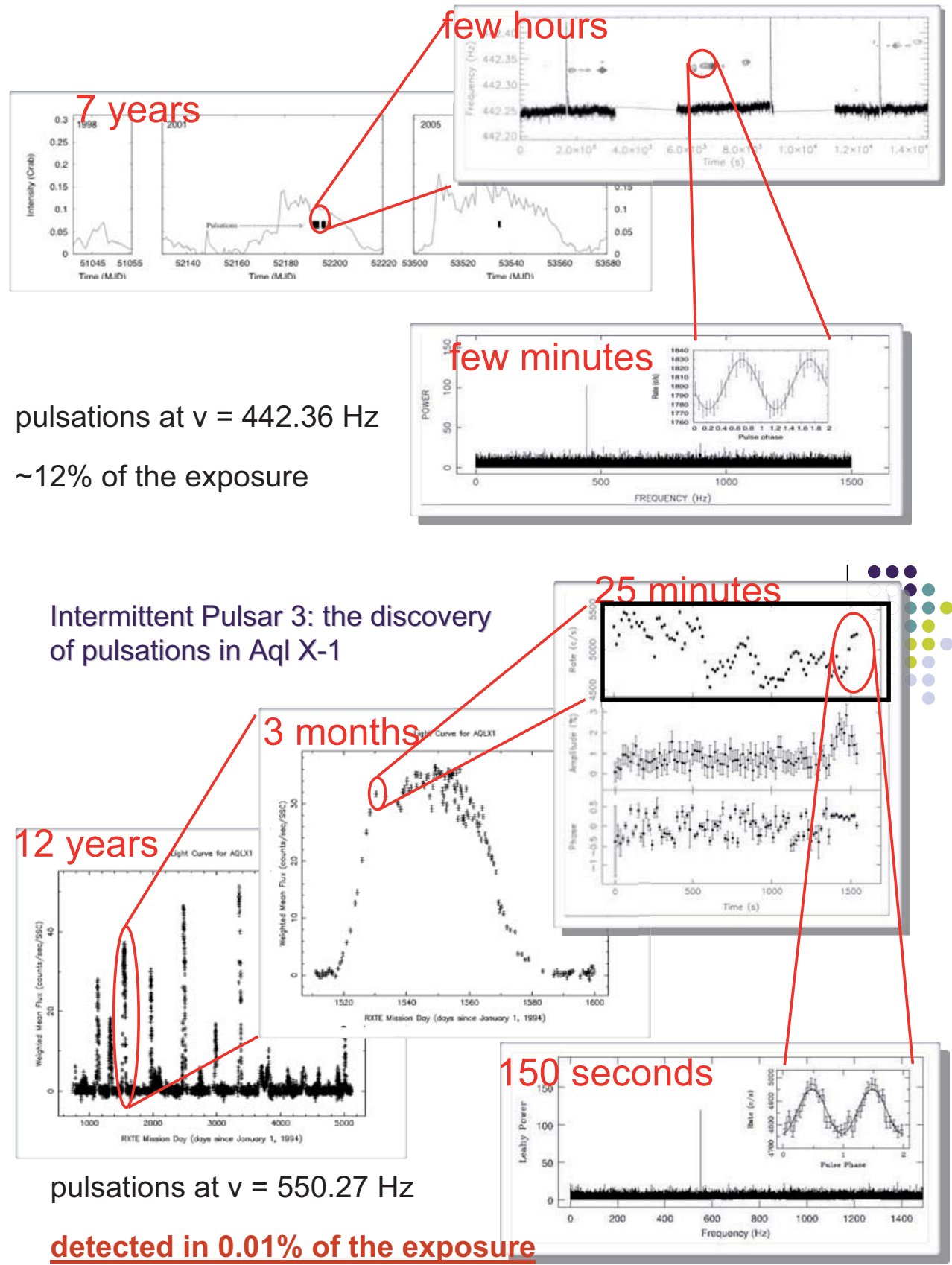


\section{The measure of the spin (part 2)}

\section{Thermonuclear explosions, a.k.a. Type I X-ray busrts}

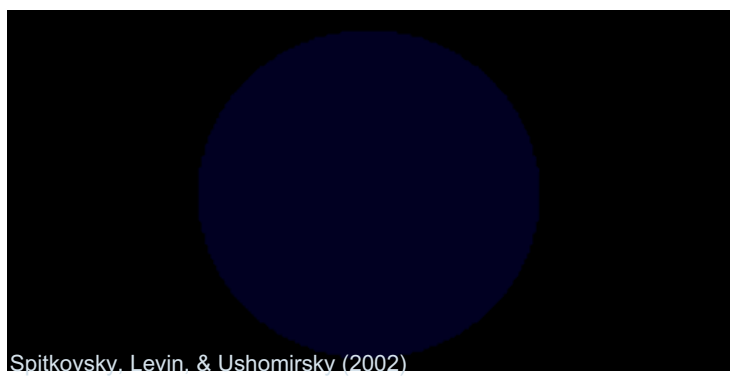

$$
\begin{aligned}
\alpha & \equiv \frac{\int L_{\mathrm{accr}} d t}{E_{\mathrm{burst}}} \approx \frac{G M / R}{E_{\mathrm{nuc}}} \\
& \approx \frac{200 \mathrm{MeV} \text { per nucleon }}{(1-5) \mathrm{MeV} \text { per nucleon }}
\end{aligned}
$$

\section{Q 5 Mev/barion Qacc 200 Mev/barion}

Burst $\rightarrow$ very rapid unstable nuclear reaction of the accreted material

It takes many hours to accumulate an thermally unstable pile of fuel

$$
\text { But only } \sim 10-100 \text { seconds to burn it ! }
$$

So the burst is triggered in one specific position on the surface (otherwise you need identical triggering conditions to better than 1 part over 1000 for the local themal instability to occur simultaneously on the whole surface) 


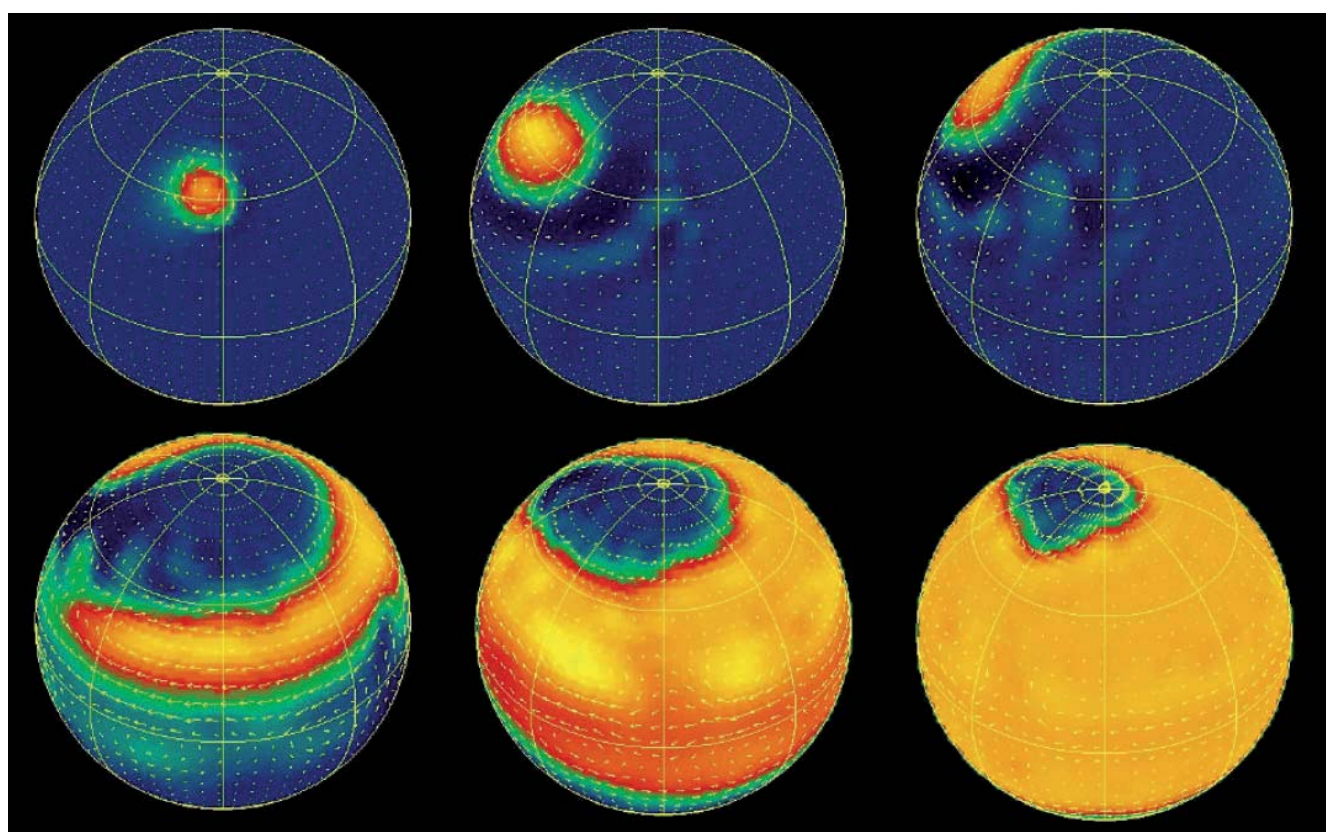

\section{Burst oscillations: nuclear powered pulsars}

SAX J1808.4-3658 confirms that the asymptotic frequency of burst oscillations is the spin frequency of the NS
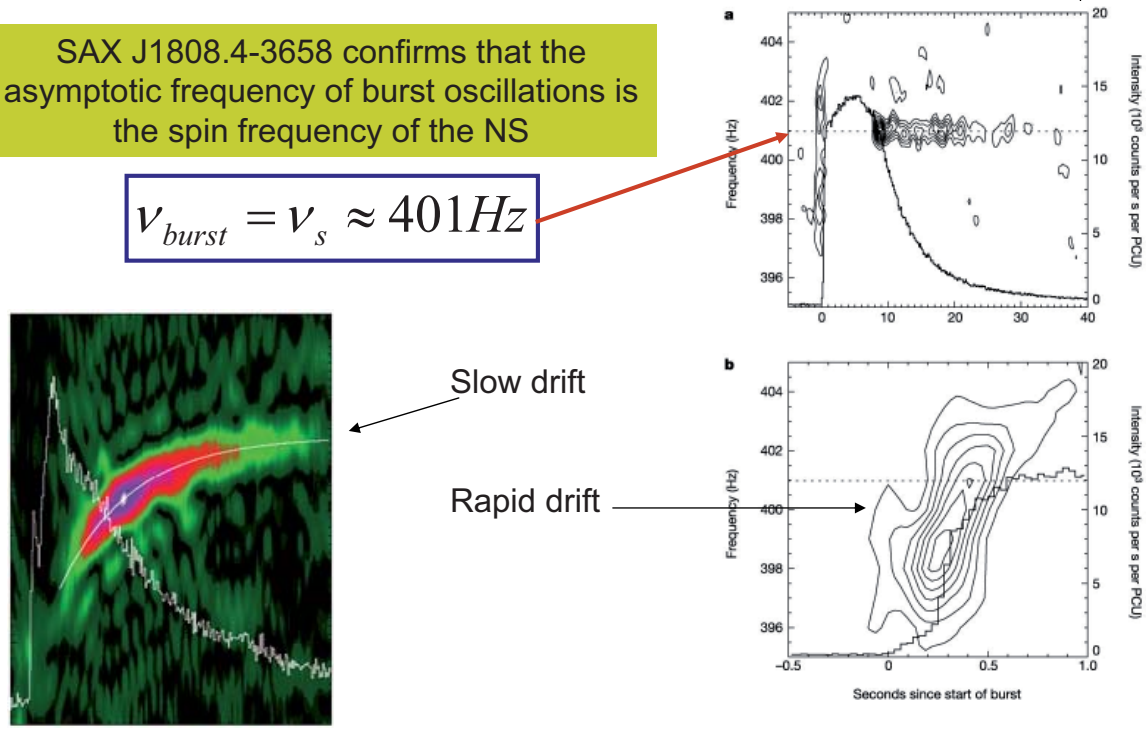


\section{Do submillisecond pulsar exist ?}

\section{What is the spin distribution of NS in LMXBs?

Nuclear powered pulsars + Accretion powered pulsars have a spin drop off at $\sim 730 \mathrm{~Hz}$

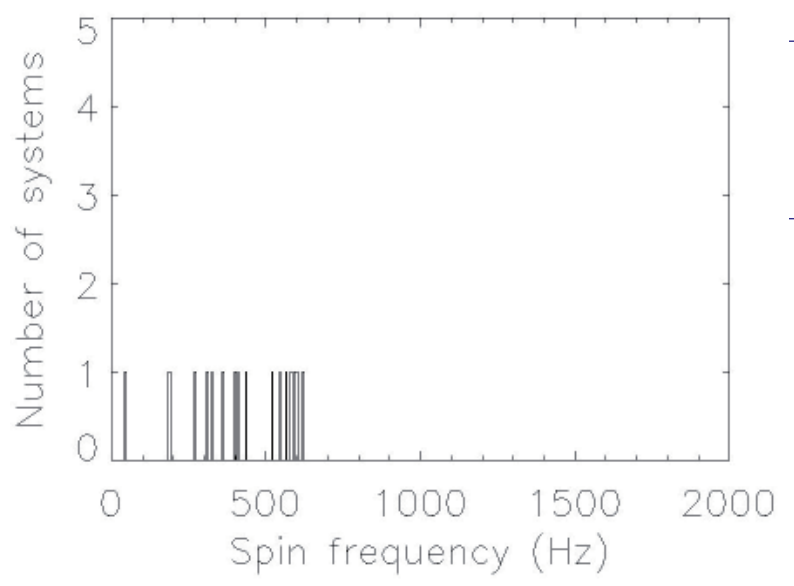

RXTE has no problem to detect a $\sim 2 \mathrm{kHz}$ oscillation. So why we don' t observe submillisecond pulsars? 


\section{Do submillisecond pulsar exist ?}

1. Steady disc accretion onto a magnetized neutron star will lead to an equilibrium period if:

$$
\begin{aligned}
& R_{A}=\left(\frac{2 \mu^{2} G^{2} M_{N S}^{2}}{\dot{M}_{c}}\right) \propto M_{N S}^{1 / 7} R^{-2 / 7} \dot{M}^{-2 / 7} \mu^{4 / 7} \\
& R_{c o}=\left(\frac{G M_{N S}}{\omega^{2}}\right)^{1 / 3} \approx 2.8 \times 10^{3} M_{N S}^{1 / 3} P_{s}^{1 / 2} K m \\
& R_{A} \sim R_{c o} \longrightarrow P_{e q}=1 s\left(\frac{B}{10^{12} G}\right)^{6 / 7}\left(\frac{\dot{M}}{10^{-9} M_{S u n} y r^{-1}}\right)^{-3 / 7}
\end{aligned}
$$

However B here is an effective field ! It's not necessarily the B field of the NS !

\section{Something more on the spin equlibrium}
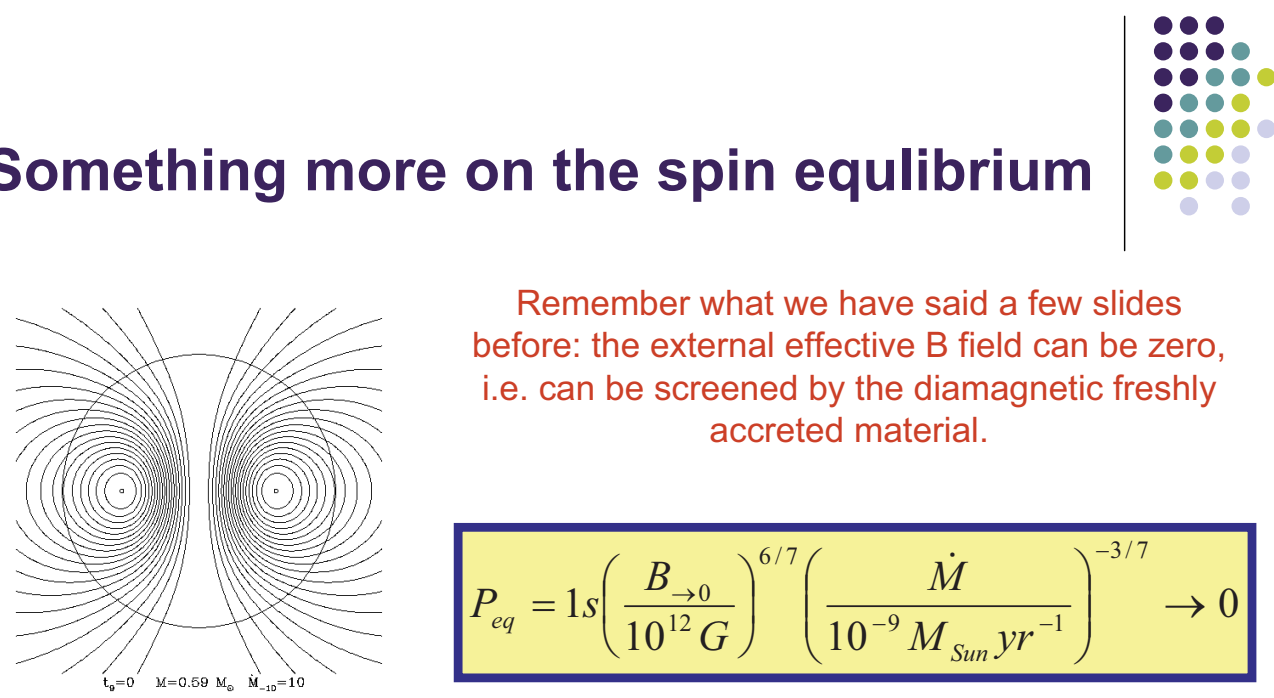

Remember what we have said a few slides before: the external effective $B$ field can be zero, i.e. can be screened by the diamagnetic freshly accreted material.

$$
P_{e q}=1 s\left(\frac{B_{\rightarrow 0}}{10^{12} G}\right)^{6 / 7}\left(\frac{\dot{M}}{10^{-9} M_{S u n} y r^{-1}}\right)^{-3 / 7} \rightarrow 0
$$

Therefore in this scenario, no limit on the equilibrium frequency exists.

So we do we observe $v_{s, \max }=716 \mathrm{~Hz} \quad$ ? 


\section{The lack of submillisecond pulsars}

1. The magnetic screening model is wrong and we don't see pulsations for another reason (e.g., intermittency)

2. The EOS forbids the spin frequency to grow above $\sim 700 \mathrm{~Hz}$ (no reasonable model can really predict that low spin frequencies)

3. The pulsar spin is blocked by another intrinsic mechanism. The best candidate is the emission of gravitational waves.

Example 1: GWs driven by r-mode instabilities can carry away substantial angular momentum

Example 2: accretion-induced crustal quadrupole moment

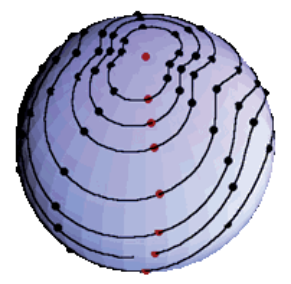

\section{Open questions for theorists (and not)}

1. What is the origin of timing noise ? Can it tell us something about the interior?

2. Why not all LMXBs pulsate ? Is possible to have an external effective $B$ field that behaves 'intermittently'?

3. Why there are no submillisecond pulsars ? Is it due to GW emission or it's a consequence of a strong $B$ field in all the NS ? 


\section{Reading}

- Romanova et al. 2008 (arXiv0803.2865R )

- Long, Romanova, Lovelace 2008

- Patruno et al. 2008

- Casella et al. 2008

- Galloway et al. 2006

- Altamirano et al. 2008

- Cumming et al. 2001

- Hartman et al. 2008

- Wijnands \& van der Klis 1998

- Chakrabarty D. 2004 (http://arxiv.org/abs/astro-ph/0408004)

- Wijnands 2006 (http://staff.science.uva.nl/ rudy/admxp/index.html)

- Lamb et al. 2008

- Watts, Patruno \& van der Klis 2008

- http://www.astro.uva.nl/xray/amxp/program.html 


\title{
Probing the neutron star physics with accreting neutron stars (part 2)
}

\author{
Alessandro Patruno \\ University of Amsterdam \\ The Netherlands
}

\section{How to probe the NS physics with NS LMXBs ?}

- X-ray spectra (cooling, cyclotron resonance, etc...)

- Coherent timing (pulse profile shape, torques, timing noise, glitches)

- Thermonuclear bursts

- Aperiodic variability (oscillation modes, QPOs)

Use of three wonderful satellites: Chandra, XMM-Newton, RXTE
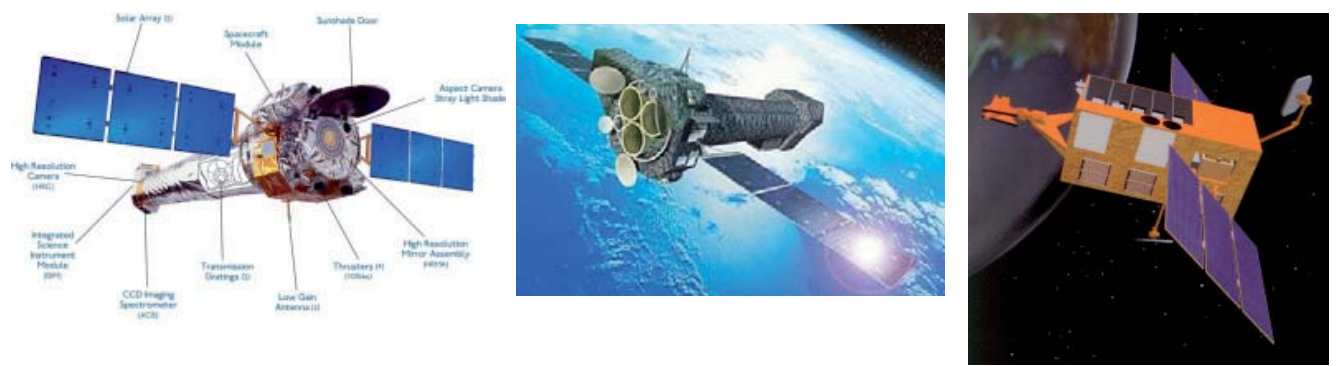


\section{Outburst vs. quiescence}

- During an outburst we observe:

1. disc + NS surface emission

2. the outburst luminosity is given by $\dot{M}_{\text {outb }}$

3. the quiescent luminosity is given by $M_{q}$

4. the average mass transfer rate is therefore: $\langle\dot{M}\rangle=\frac{\dot{M}_{\text {out }} \cdot t_{\text {out }}+\dot{M}_{q} \cdot t_{q}}{t_{q}+t_{\text {out }}}$

So we need to measure four observables (assuming $L$ and Mdot are related) to determine the average mass transfer rate

Typical outburst X-ray luminosity: $~ 1 e 36-1 e 37 \mathrm{erg} / \mathrm{s}$

Typical quiescent $X$-ray luminosity: $\sim 1 e 33 \mathrm{erg} / \mathrm{s}$

\section{The quiescent emission}

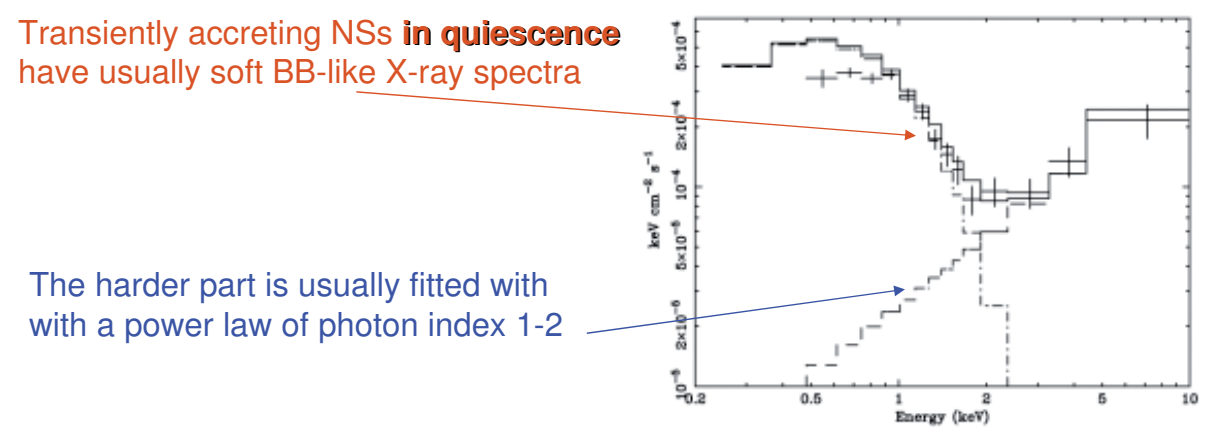

INTERPRETATION:

Black body-like component comes from the heat released from the NS surface Power law component is of unknown origin and remains unexplained (continued accretion, shock from a pulsar wind, others) 


\section{How to fit a quiescent spectrum ? BB vs. NSA models}

The spectrum of a NS is not a pure BB for two reasons:

2. There is an atmosphere with a chemical composition, a magnetic field.

3. The free free absorption (absorption of a photon by the free electron in the Coulomb field of a ion) is proportional to $v^{-3}$
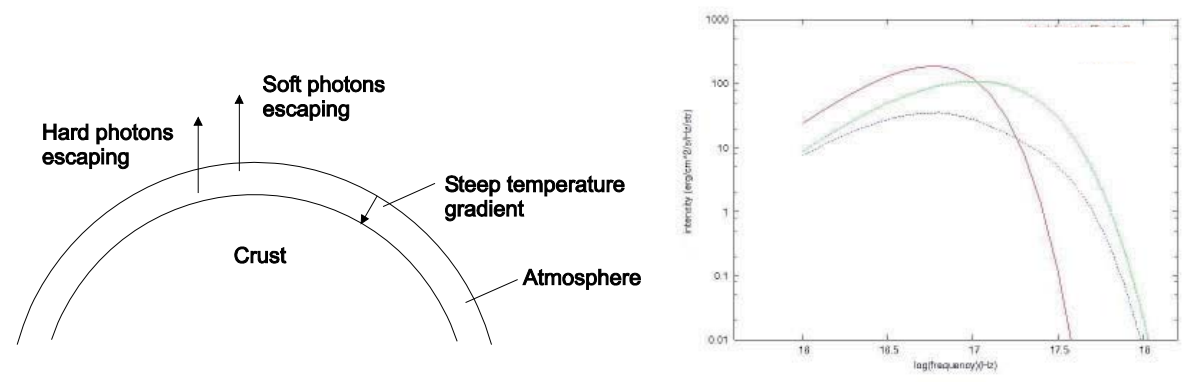

\section{Heating and cooling of NSs}

The accreted material sinks to a depth of $\sim 900 \mathrm{~m}$ and then burns via pycnonuclear reactions and

Incandescent luminosity:

$$
\text { beta captures }
$$

$$
\begin{aligned}
& L_{i} \approx f Q_{n u c}\left(\frac{1}{t_{r}} \int \dot{M} d t\right) \equiv f Q_{n u c}\langle\dot{M}\rangle \\
& Q_{n u c} \approx 1-1.5 \mathrm{MeV} / m_{p}
\end{aligned}
$$

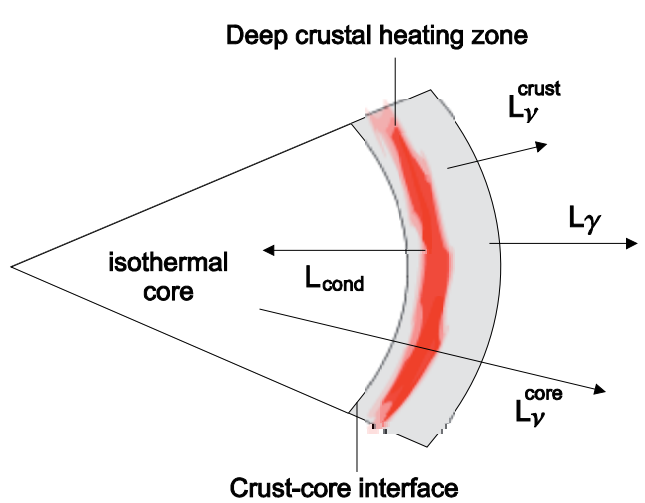




\section{The crust-core coupling}

- A fraction $f$ or heat flows into the core

- A fraction 1-f flows into the crust

The core has high thermal conductivity and heat capacity $\rightarrow$ temperature is almost unchanged The crust has high thermal conductivity and low heat capacity $\rightarrow$ temperature significantly incresed by the heat flow

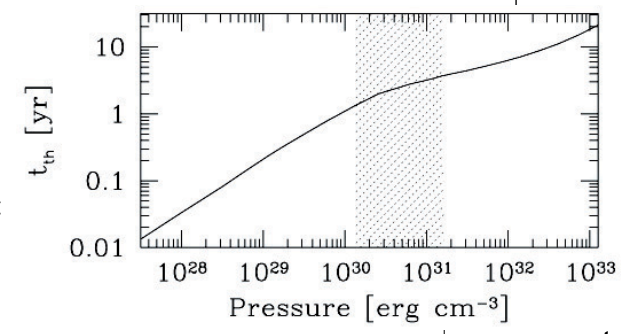

$$
\begin{gathered}
\frac{L_{o} t_{o}}{L_{i} t_{r}}=\frac{G M / R}{f Q_{\text {nucl }}} \approx \frac{200}{f} \\
t_{\text {th }}=\frac{1}{4}\left[\int_{0}^{P}\left(\frac{c_{P}}{K \rho}\right)^{1 / 2} \frac{d P}{g}\right]
\end{gathered}
$$

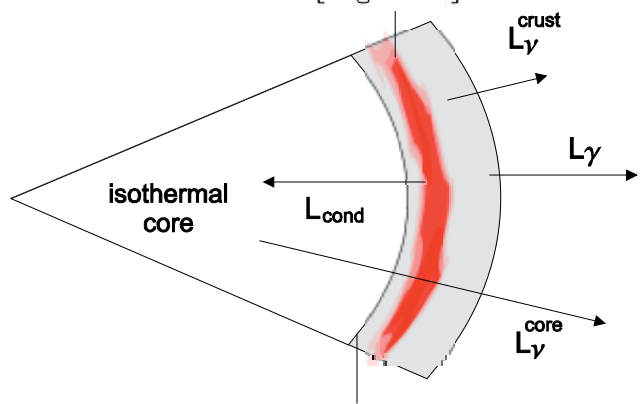

Crust-core interface

\section{The quasi persistent transients}

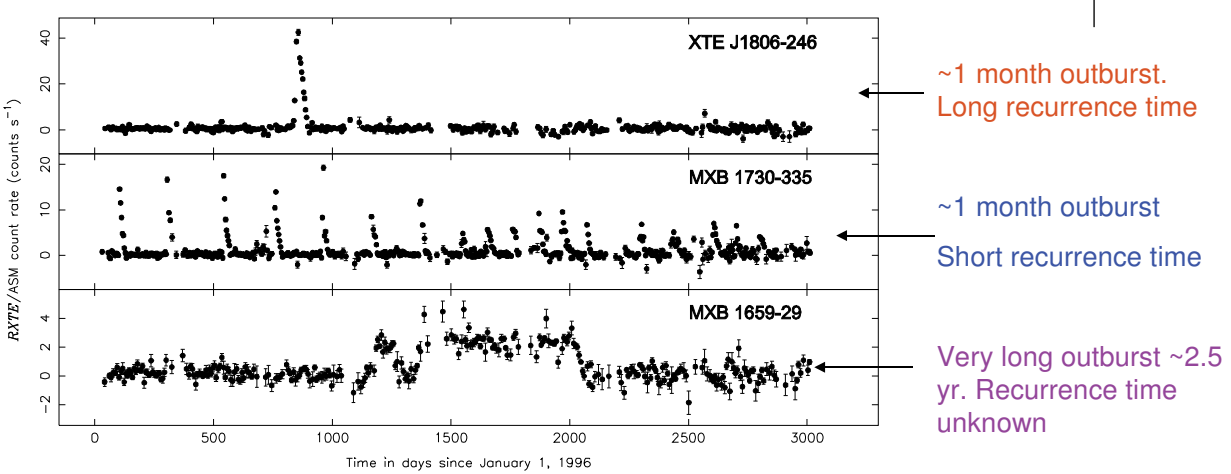

Two transient LMXBs show very long outbursts with length of the order

of $\sim 1-10 \mathrm{yr}$. This means that the quiescent luminosity is very high with respect to the normal transients with outburst length of $\sim 1$ month

Deep crustal heating can thus break the core-crust coupling and make the crust much hotter than the core 


\section{How many quasi persistent transients do we know?}

\begin{tabular}{|l|l|}
\hline Source name & StatuS \\
\hline EXO 0748-676 & $\begin{array}{l}\text { Detected in outburst since } \\
\text { February } 1985\end{array}$ \\
\hline GS 1826-238 & $\begin{array}{l}\text { Detected in outburst since } \\
\text { September 1988 }\end{array}$ \\
\hline XTE J1759-220 & $\begin{array}{l}\text { Detected in outburst since } \\
\text { February } 2001\end{array}$ \\
\hline 4U 2129+47 & $\begin{array}{l}\text { Quiescent since 1983 after at } \\
\text { least 11 years in outburst }\end{array}$ \\
\hline X 1732-304 & $\begin{array}{l}\text { Quiescent since 1999 after at } \\
\text { least 12 years in outburst }\end{array}$ \\
\hline KS 1731-260 & $\begin{array}{l}\text { Turned off in February 2001 } \\
\text { after an outburst of } ~ 12.5 \text { years }\end{array}$ \\
\hline MXB 1659-29 & $\begin{array}{l}\text { Turned off in Spetember 2001 } \\
\text { after an outburst of } ~ 2.5 \text { years }\end{array}$ \\
\hline
\end{tabular}

\section{KS 1731-260}

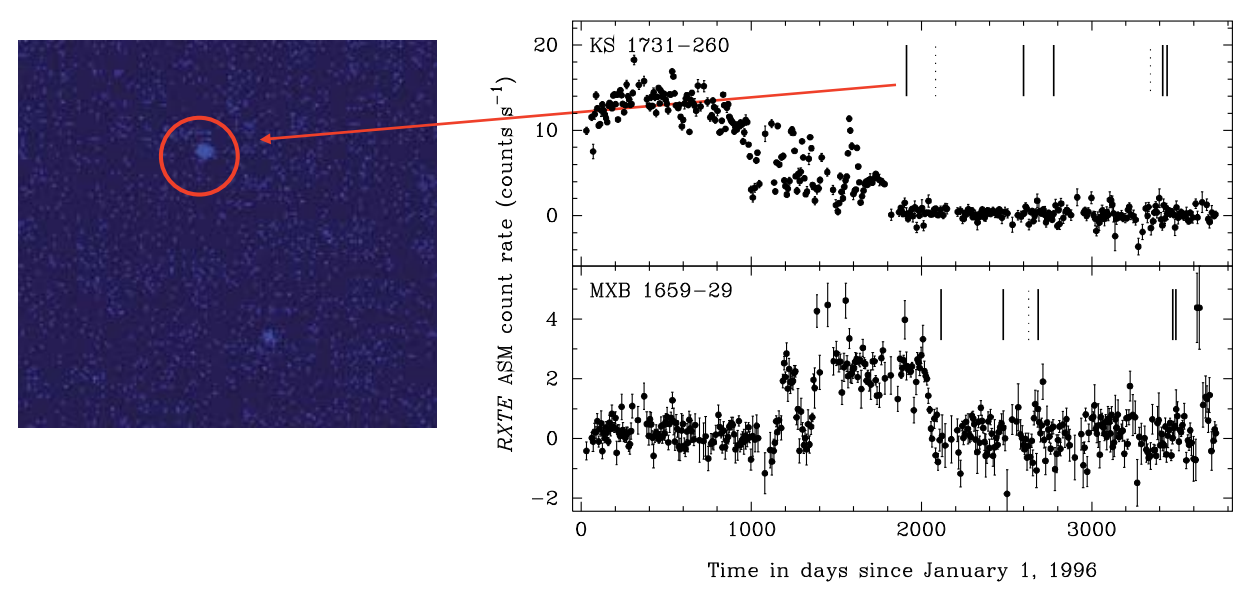

In quiescence all the LMXBs are very faint ! Luminosities of $\sim 1 \mathrm{e} 32-1 \mathrm{e} 33 \mathrm{erg} / \mathrm{s}$ 


\section{MXB 1659-29}

Very recent new Chandra observation on 2008 Apr. 27

The total quiescent monitoring now extends up to $6.6 \mathrm{yrs}$

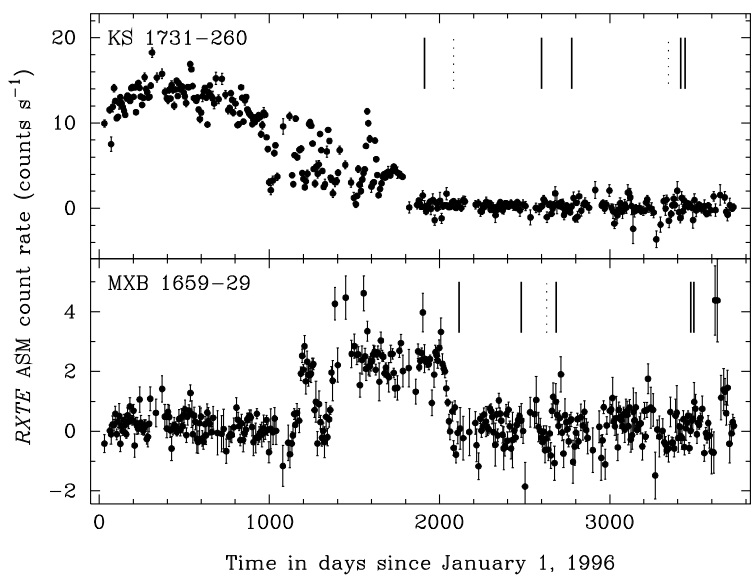

\section{Power law vs. exponential decay: the situation 'till early 2008}
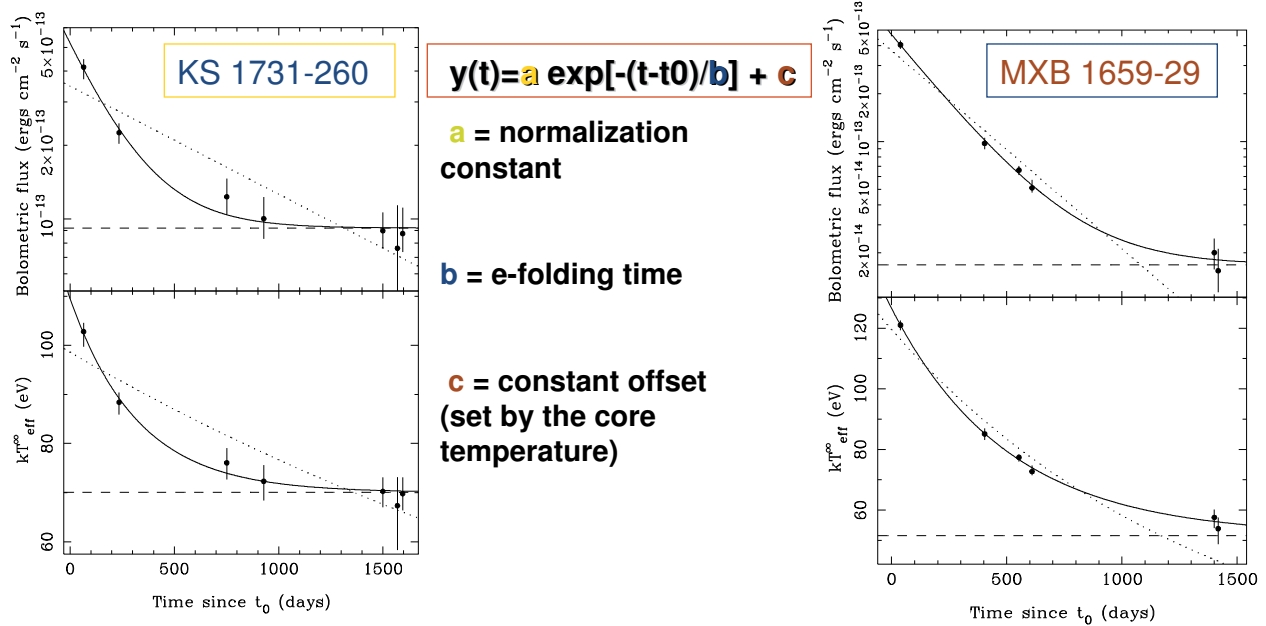

Flux and Temperature well fitted by an exponential decay plus a constant offset (set by the core temperature) 


\section{The thermal relaxation timescale and the surface temperature}

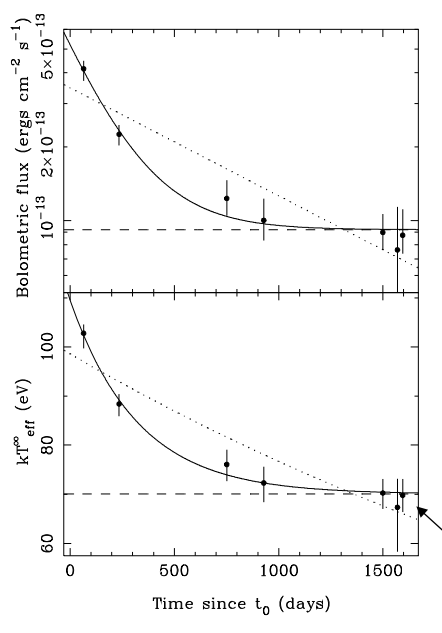

In KS 1731 we have not

reached the equilibrium between the core and the crust yet.

The constant flux level indicates a 70(2) eV surface temperature and an e-folding timescale of $325(101)$

Some residual slope

is still possible

\section{The new observation of MXB 1659-29}

Before April 2008
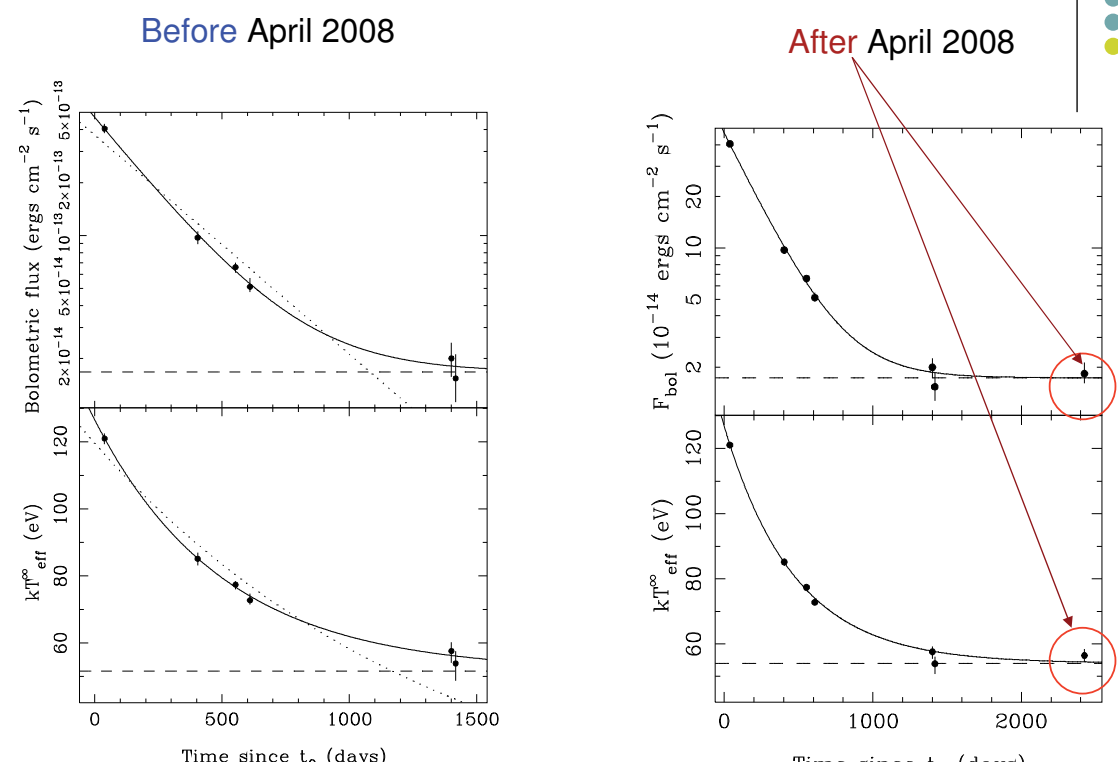

Power law model does not fit the data! 


\section{New constraints for MXB 1659}

\begin{tabular}{|l|l|l|l|l|}
\hline & NSA (D=10 kpc) & NSA (D=5kpc) & NSA (D=13kpc) & BB \\
\hline $\begin{array}{l}\text { Normalization } \\
(\mathrm{a}, \mathrm{eV})\end{array}$ & $73(2)$ & $54(1)$ & $82(2)$ & $176(11)$ \\
\hline $\begin{array}{l}\text { e-folding time } \\
\text { (b, days) }\end{array}$ & $472(23)$ & $485(27)$ & $473(24)$ & $437(43)$ \\
\hline $\begin{array}{l}\text { Constant level } \\
(\mathrm{c}, \mathrm{eV})\end{array}$ & $54(1)$ & $45(1)$ & $58(1)$ & $141(3)$ \\
\hline
\end{tabular}

\section{How model dependent is the result?}

1. e-folding timescales are consistent with each other with any model assumed

2. Shape of the cooling curve independent from the distance

3. Core temperature can be inferred from the relaxed surface emission, by integrating the thermal structure of the crust.

4. Core temperature: $3.5 \times 10^{\wedge} 7 \mathrm{~K}(\mathrm{kT} \sim 7 \mathrm{keV})$ deep He layer overlying a pure Fe layer)

$$
\begin{aligned}
& 8.3 \times 10^{\wedge} 7 \mathrm{~K}(\mathrm{kT} \sim 3 \mathrm{keV}) \text { shallow He layer overlying a layer of heavy } \\
& \mathrm{rp} \text {-process ashes }
\end{aligned}
$$

Modified URCA predicts:

$$
2 \times 10^{29} \mathrm{erg} / \mathrm{s}<L_{v}<2 \times 10^{32} \mathrm{erg} / \mathrm{s}
$$

Incandescent luminosity observed (for $\mathrm{D}=10 \mathrm{kpc}$ ) $L_{i} \approx 6 \times 10^{33}$

Therefore even in the most optimistic case there is a factor 30 in difference between what predicted by the minimal cooling paradigm and the observed luminosity 


\section{Enhanced neutrino \& high thermal conductivity of the crust?}

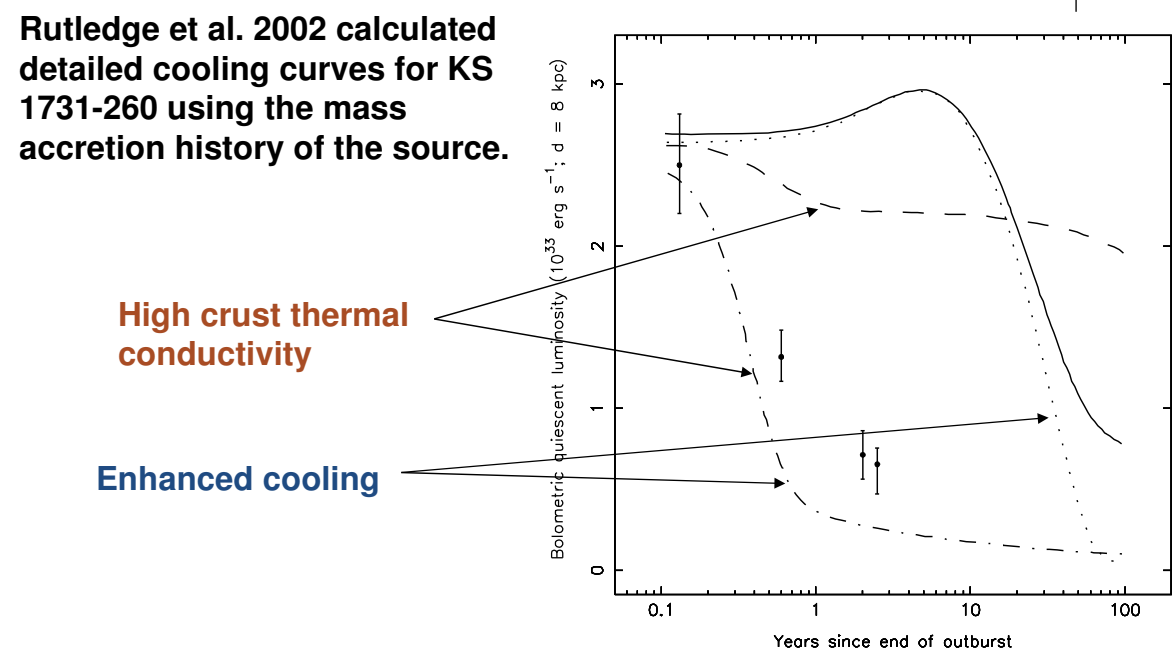

\section{Enhanced neutrino \& high thermal conductivity of the crust?}

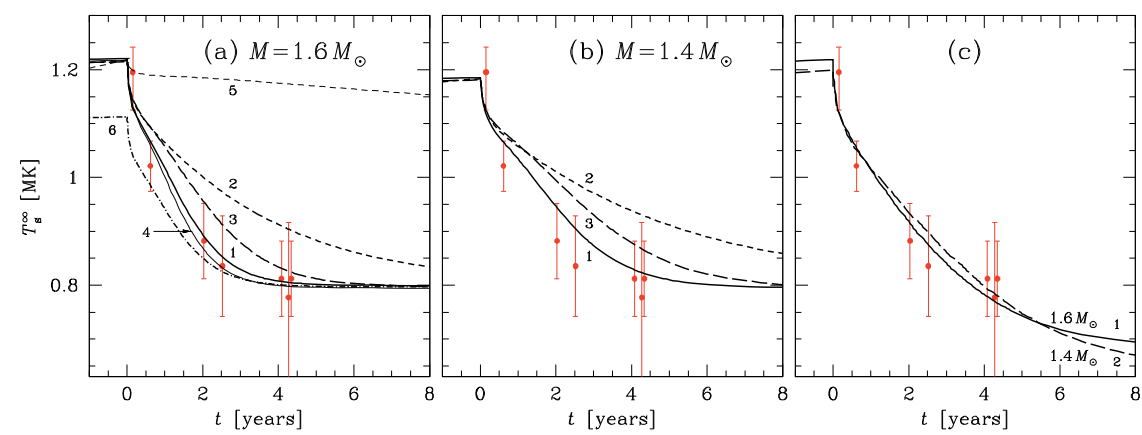

With the current observation we can't confirm (yet ?) that KS 1731-260 requires enhanced cooling emission. It can be fit with a power law model or an exponential decay equally well. The only requirement is an high thermal conductivity of the crust

Beta capture can produce nuclei in excited states $\rightarrow$ deexcitation can generate extra heat $\rightarrow$ no enhanced cooling required 


\section{Exponential vs. power law}

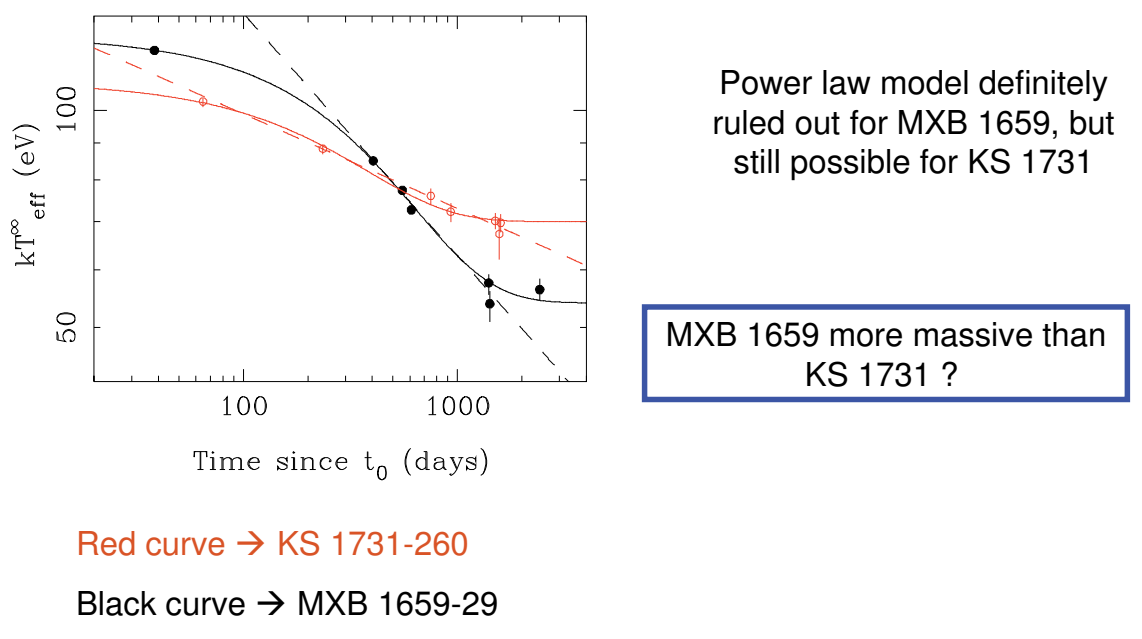

\section{SAX J1808.4-3658}

Outbursts last for $\sim 1$ month

Recurrence time quite well known: $2.5 \mathrm{yr}$ (observed outbursts in the 1996, 1998, 2000, 2002, 2005)

Low magnetic field: B 1e8 G Distance of approx. 2.5 -- $3.5 \mathrm{kpc}$

Very low luminosity in quiescence: $~ 5 e 31 \mathrm{erg} / \mathrm{s}$

Known mass transfer rate: Mdot 1e-10 Msun/yr

ONE OF THE BEST KNOWN LMXBS!

-Pulsations

-- Thermonuclear bursts

-- Bursts oscillations

-- Twin kHz QPOs

-- Fast cooling

-- Multiple outbursts

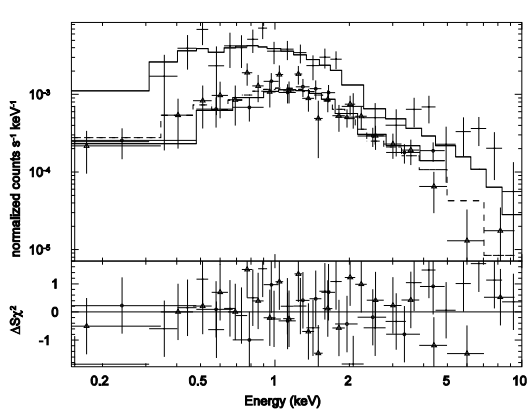




\section{Minimal cooling paradigm}

Note: the problem here is different ! We're not trying to measure the surface temperature evolution with time, we are trying to observe the

minimum luminosity of the source for a given mass transfer rate

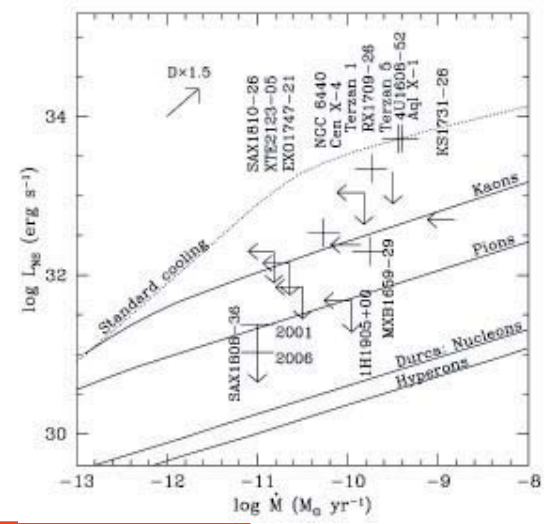

Quasi persistent sources (KS 1731, MXB 1659) are HOT, and emit a HIGH flux in the early stages of quiescence

$$
L_{\text {bol }} \approx 10^{33} \mathrm{erg} / \mathrm{s}
$$
How fast does it $\mathrm{cool}$ ?

Normal transients (SAX J1808.4) can be COLD and emit a LOW flux during quiescence $L_{b o l} \approx 5 \times 10^{31} \mathrm{erg} / \mathrm{s}$

\section{Minimal cooling paradigm}

\begin{tabular}{|l|l|l|l|}
\hline Epoch & $\begin{array}{l}\mathrm{NH} \\
(1 \mathrm{e} 22 \\
\left.\mathrm{cm}^{\wedge}-2\right)\end{array}$ & $\begin{array}{l}\mathrm{kT} \\
(\mathrm{eV})\end{array}$ & $\begin{array}{l}\mathrm{L} \\
(\mathrm{erg} / \mathrm{s})\end{array}$ \\
\hline 2001 & 0.13 & $<42$ & $2.4 \mathrm{e} 31$ \\
\hline 2006 & 0.13 & $<35$ & $1.2 \mathrm{e} 31$ \\
\hline $\begin{array}{l}2001 \& \\
2006\end{array}$ & 0.13 & $<34$ & $1.1 \mathrm{e} 31$ \\
\hline $\begin{array}{l}2001 \& \\
2006\end{array}$ & $0.15(4)$ & $<61$ & $1.0 \mathrm{e} 31$ \\
\hline
\end{tabular}

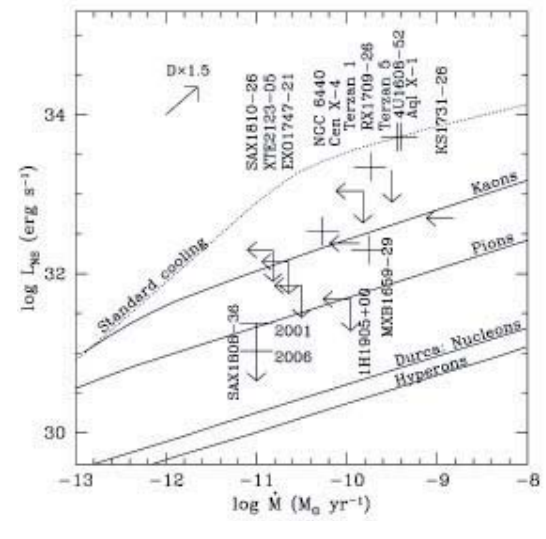




\section{Sources of error}

- Distance $\mathrm{D}=3.5(1) \mathrm{kpc} \rightarrow 6 \%$ uncertainity

- Mass and radius $\rightarrow 3 \%(M=1.4 \mathrm{R}=10 \mathrm{Km}$ to $\mathrm{M}=2.0 \mathrm{R}=12 \mathrm{Km})$

- Mass transfer rate assumed to be the observed one

Assuming 50\% uncertainty in mass transfer rate and distance still requires enhanced cooling for SAX J1808. Observations need to be highly biased from an unknown source of error to move SAX J1808 from the enhanced cooling region

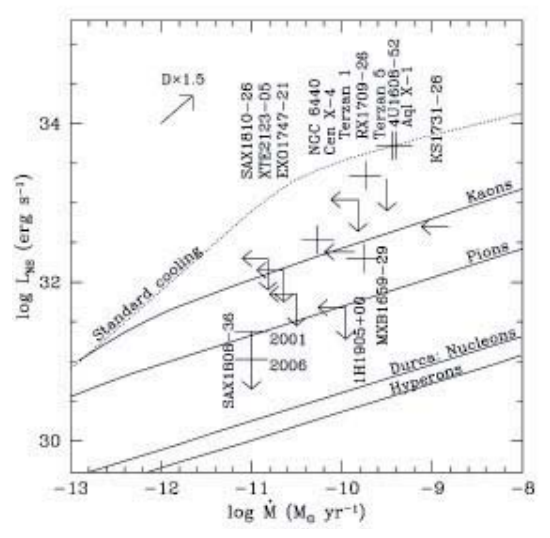

\section{Why the thermal component is not residual accretion?}

- Accretion shows variability on short timescale while we see a smooth exponential decay

Therefore the surface emission is quite robust

- If residuals accretion takes place, we expect variation on the observed quiescent luminosity from cycle to cycle

Major sources of uncertainity:

2. Distance (and therefore the X-ray Luminosity)

3. Recurrence time (and therefore the AVERAGE mass transfer rate) 


\section{Reading}

- Yakovlev \& Pethick

- Page, Geppert \& Weber

- Cackett et al.

- Chackett et al.

- Brown \& Bildsten

- Rutledge et al.

- Heinke et al.

- More references will appear later...check on the website 